\title{
The Linear Dynamic Complementarity Problem is a special case of the Extended Linear Complementarity Problem*
}

\author{
B. De Schutter and B. De Moor
}

If you want to cite this report, please use the following reference instead: B. De Schutter and B. De Moor, "The Linear Dynamic Complementarity Problem is a special case of the Extended Linear Complementarity Problem," Systems \& Control Letters, vol. 34, no. 1-2, pp. 63-75, May 1998. 


\title{
The Linear Dynamic Complementarity Problem is a special case of the Extended Linear Complementarity Problem
}

\author{
B. De Schutter ${ }^{1}$ B. De Moor ${ }^{2}$ \\ ESAT-SISTA, K.U.Leuven, Kardinaal Mercierlaan 94, B-3001 Heverlee (Leuven), \\ Belgium, email: bart.deschutter@esat.kuleuven.ac.be, \\ bart.demoor@esat.kuleuven.ac.be
}

\begin{abstract}
In this paper we consider some extensions of the Linear Complementarity Problem, which is one of the fundamental problems in mathematical programming. More specifically we consider the Linear Dynamic Complementarity Problem (LDCP), the Generalized Linear Complementarity Problem (GLCP) and the Extended Linear Complementarity Problem (ELCP). We show that the LDCP is a special case of the ELCP and of the GLCP.
\end{abstract}

Key words: linear complementarity problem, extended linear complementarity problem, generalized linear complementarity problem, discrete event system, hybrid system

\section{Introduction}

In this paper we consider three extensions of the Linear Complementarity Problem, which is one of the fundamental problems in mathematical programming.

The Linear Dynamic Complementarity Problem (LDCP) has been introduced by J.M. Schumacher in [14] where it has been used to determine the uniqueness of smooth continuations and the associated mode selection problem for a class of systems with discontinuous dynamics. In $[4,5]$ De Moor introduced

$\overline{1}$ Senior research assistant with the F.W.O. (Fund for Scientific Research - Flanders)

2 Research associate with the F.W.O. 
the Generalized Linear Complementarity Problem, which can be used to determine operating points and transfer characteristics of piecewise-linear resistive electrical circuits. In $[6,7]$ we have presented another extension of the LCP, the Extended Linear Complementarity Problem (ELCP). The ELCP can be used to solve some fundamental problems in the max-plus algebra, which is one of the frameworks that can be used to model a class of discrete event systems. Furthermore, in $[10,11]$ we have used the ELCP to compute optimal traffic light control schemes for a single intersection.

In this paper we show that the LDCP is a special case of the ELCP and of the GLCP.

All the systems mentioned above (systems with discontinuous dynamics, piecewise-linear resistive electrical circuits, discrete event systems and traffic systems) can be considered as special cases of hybrid systems. Since the ELCP appears in one form or another in the analysis of these systems, this seems to indicate that the ELCP may play an important role as a kind of generalized framework in the analysis of many classes of hybrid systems.

\section{Notations}

All the vectors that appear in this paper are assumed to be column vectors, i.e., matrices with one column. If $a$ is a vector then $a_{i}$ represents the $i$ th component of $a$. The $n$ by $n$ identity matrix is represented by $I_{n}$ and the $m$ by $n$ zero matrix by $O_{m \times n}$. If the dimensions of the identity matrix or the zero matrix are clear from the context they will be omitted.

If $a$ is a vector with $n$ components then $a \geq 0$ means that $a_{i} \geq 0$ for $i=$ $1,2, \ldots, n$. Likewise $a=0$ means that $a_{i}=0$ for $i=1,2, \ldots, n$.

A vector $a \in \mathbb{R}^{n}$ is lexicographically nonnegative, denoted by $a \succeq 0$, if either $a_{i}=0$ for all $i$ or the first nonzero component of $a$ is positive.

If $a \in \mathbb{R}^{n}$ then the sign decomposition $a^{+}, a^{-}$of $a$ is defined as follows: $a^{+}, a^{-} \in$ $\mathbb{R}^{n}, a=a^{+}-a^{-}$with $a^{+}, a^{-} \geq 0$ and $\left(a^{+}\right)^{T} a^{-}=0$.

If $\mathcal{A}$ is a set then $\# \mathcal{A}$ is the cardinality of $\mathcal{A}$. 


\section{The Linear Complementarity Problem and some of its extensions}

\subsection{The Linear Complementarity Problem}

One of the possible formulations of the Linear Complementarity Problem (LCP) is the following [2]:

Given $M \in \mathbb{R}^{n \times n}$ and $q \in \mathbb{R}^{n}$, find $w, z \in \mathbb{R}^{n}$ such that

$$
\begin{aligned}
w, z & \geq 0 \\
w & =q+M z \\
z^{T} w & =0
\end{aligned}
$$

or show that no such $w$ and $z$ exist.

Note that if $w$ and $z$ are solutions of the LCP then it follows from (1) and (3) that $z_{i} w_{i}=0$ for $i=1,2, \ldots, n$. So for each index $i \in\{1,2, \ldots, n\}$ at least one of the following conditions should hold:

$$
\begin{array}{lll}
z_{i}=0 & \text { and } & w_{i} \geq 0 \\
z_{i} \geq 0 & \text { and } & w_{i}=0
\end{array}
$$

Hence, we have

$$
w_{i}>0 \Rightarrow z_{i}=0 \quad \text { and } \quad z_{i}>0 \Rightarrow w_{i}=0 \quad \text { for } i=1,2, \ldots, n,
$$

i.e., the zero patterns of $w$ and $z$ are complementary. Therefore, condition (3) is called the complementarity condition of the LCP.

The LCP has numerous applications such as quadratic programming problems, determination of the Nash equilibrium of a bimatrix game problem, the market equilibrium problem, the optimal invariant capital stock problem, the optimal stopping problem, etc. [2]. This makes the LCP one of the fundamental problems of mathematical programming. For more information on the $\mathrm{LCP}$ and its applications the interested reader is referred to $[2,12]$.

\subsection{The Linear Dynamic Complementarity Problem}

The Linear Dynamic Complementarity Problem (LDCP) is defined as follows [14-16]:

Given matrices $A \in \mathbb{R}^{n \times n}, B \in \mathbb{R}^{n \times k}, C \in \mathbb{R}^{k \times n}$ and $D \in \mathbb{R}^{k \times k}$, find for a given $x_{0} \in \mathbb{R}^{n}$ sequences $\left\{y_{l}\right\}_{l=0}^{n-1},\left\{u_{l}\right\}_{l=0}^{n-1}$ with $y_{l}, u_{l} \in \mathbb{R}^{k}$ for all $l$ such that 


$$
\begin{aligned}
y_{0} & =C x_{0}+D u_{0} \\
y_{1} & =C A x_{0}+C B u_{0}+D u_{1} \\
& \vdots \\
y_{n-1} & =C A^{n-1} x_{0}+C A^{n-2} B u_{0}+\ldots+C B u_{n-2}+D u_{n-1}
\end{aligned}
$$

and such that for each index $i \in\{1,2, \ldots, k\}$ at least one of the following statements is true:

$$
\begin{aligned}
& {\left[\begin{array}{llll}
\left(y_{0}\right)_{i} & \ldots & \left(y_{n-1}\right)_{i}
\end{array}\right]^{T}=0 \text { and }\left[\begin{array}{lll}
\left(u_{0}\right)_{i} & \ldots & \left(u_{n-1}\right)_{i}
\end{array}\right]^{T} \succeq 0} \\
& {\left[\begin{array}{lll}
\left(y_{0}\right)_{i} & \ldots & \left(y_{n-1}\right)_{i}
\end{array}\right]^{T} \succeq 0 \text { and }\left[\left(u_{0}\right)_{i} \ldots\left(u_{n-1}\right)_{i}\right]^{T}=0 .}
\end{aligned}
$$

Conditions (9) - (10) are called the complementarity conditions of the LDCP since they resemble the complementarity conditions (4)-(5) of the LCP.

In $[14-16]$ the LDCP has been used to determine the uniqueness of smooth continuations and the associated mode selection problem for a class of systems with discontinuous dynamics - the "complementary-slackness systems", typical examples of which are electrical networks with diodes and mechanical systems subject to geometric inequality constraints.

In $[14,15]$ it has been shown that under fairly mild assumptions ${ }^{3}$ the LDCP can be reduced to a series of LCPs.

\subsection{The Generalized Linear Complementarity Problem}

In $[4,5]$ De Moor introduced the following extension of the LCP:

Given $Z \in \mathbb{R}^{p \times n}$ and $m$ subsets $\phi_{1}, \phi_{2}, \ldots, \phi_{m}$ of $\{1,2, \ldots, n\}$, find a nontrivial $u \in \mathbb{R}^{n}$ such that

$$
\sum_{j=1}^{m} \prod_{i \in \phi_{j}} u_{i}=0
$$

subject to $u \geq 0$ and $Z u=0$.

In $[4,5]$ this problem is called the Generalized Linear Complementarity Problem (GLCP). It is easy to verify that the LCP is a special case of the GLCP. Condition (11) is called the complementarity condition of the GLCP.

In $[4,17]$ it has been shown that the GLCP can be used to determine operating points and transfer characteristics of piecewise-linear resistive electrical circuits.

$\overline{3}$ I.e., if for some $j \in \mathbb{N}$ we have $D=C B=\ldots=C A^{j-1} B=O$ and the principal minors of $C A^{j} B$ are positive. 


\subsection{The Extended Linear Complementarity Problem}

\subsubsection{Definition}

Another extension of the LCP, the Extended Linear Complementarity Problem (ELCP), is defined as follows $[6,7]$ :

Given $A \in \mathbb{R}^{p \times n}, B \in \mathbb{R}^{q \times n}, c \in \mathbb{R}^{p}, d \in \mathbb{R}^{q}$ and $m$ subsets $\phi_{1}, \phi_{2}, \ldots, \phi_{m}$ of $\{1,2, \ldots, p\}$, find $x \in \mathbb{R}^{n}$ such that

$$
\sum_{j=1}^{m} \prod_{i \in \phi_{j}}(A x-c)_{i}=0
$$

subject to $A x \geq c$ and $B x=d$.

Condition (12) is the complementarity condition of the ELCP. Note that this condition is equivalent to

$$
\forall j \in\{1,2, \ldots, m\}, \exists i \in \phi_{j} \text { such that }(A x-c)_{i}=0 \text {. }
$$

So we could say that each set $\phi_{j}$ corresponds to a group of inequalities of $A x \geq c$ and that in each group at least one inequality should hold with equality.

In $[6,8,9]$ we have shown that the ELCP can be used to solve many problems that arise in the system theory for max-linear time-invariant discrete event systems, i.e., discrete event systems that can be described by a time-invariant model that is linear in the max-plus algebra [1,3], which has maximization and addition as its basic operations.

\subsubsection{Homogenization}

Suppose that we want to solve the system $B x=d$. This system can be made homogeneous as follows. We introduce a parameter $\alpha$ and solve the equivalent homogeneous system $C y=0, \alpha \neq 0$ with $C=\left[\begin{array}{ll}B & -d\end{array}\right]$ and $y=\left[\begin{array}{ll}x^{T} & \alpha\end{array}\right]^{T}$. It is easy to verify that every solution of $B x=d$ corresponds to a solution $\left[\begin{array}{ll}x^{T} & 1\end{array}\right]^{T}$ of $C y=0$. Moreover, every solution of $C y=0$ with a nonzero $\alpha$ component can be divided by the $\alpha$ component, which leads to a solution of the form $\left[\begin{array}{ll}x^{T} & 1\end{array}\right]^{T}$ of $C y=0$, and clearly $x$ is a solution of $B x=d$. Note that we may assume without loss of generality that $\alpha$ is nonnegative.

Using homogenization the general ELCP defined in Section 3.4.1 can be transformed into an homogeneous ELCP. In general the homogeneous ELCP is 
defined as follows:

Given $P \in \mathbb{R}^{p \times n}, Q \in \mathbb{R}^{q \times n}$ and $m$ subsets $\phi_{1}, \phi_{2}, \ldots, \phi_{m}$ of $\{1,2, \ldots, p\}$, find a non-trivial vector $u \in \mathbb{R}^{n}$ such that

$$
\sum_{j=1}^{m} \prod_{i \in \phi_{j}}(P u)_{i}=0
$$

subject to $P u \geq 0$ and $Q u=0$, or show that no such $u$ exists.

It can be shown that the ELCP is a generalization of the GLCP and that the homogeneous ELCP and the GLCP are equivalent [6,7]. In [4] De Moor has developed an algorithm to compute the complete solution set of a GLCP. In $[6,7]$ we have extended this algorithm in order to compute the complete solution set of an ELCP.

\subsubsection{Complexity issues}

Although the general ELCP can be transformed into an homogeneous ELCP and although the homogeneous ELCP and the GLCP are equivalent, it is not recommended to use the GLCP algorithm of [4] to solve an ELCP since this approach has a few drawbacks:

- To convert the homogeneous ELCP into a GLCP we have to introduce some additional variables $[6,7]$, which increases the complexity of the problem. Since the execution time of the algorithm of [4] increases rapidly as the number of unknowns increases, it is certainly not advantageous to have a large number of variables.

- Moreover, using the algorithm of [4] to compute the solution set of an ELCP will in general result in a redundant description of the solution set of the ELCP.

The ELCP algorithm of [6,7] will yield a concise description of the solution set of an ELCP and it will also be much faster than the algorithm that uses the transformation into a GLCP.

In $[6,7]$ we have shown that the general ELCP with rational data is an NPhard problem. Moreover, the execution time of the ELCP algorithm of [6,7], which computes a description of the complete solution set of an ELCP, depends polynomially on the number of (in)equalities and exponentially on the number of variables. 


\section{The LDCP is a special case of the ELCP and the GLCP}

\subsection{Composing complementarity conditions of GLCPs and ELCPs}

In this section we show that composing complementarity conditions of a GLCP by logical operators such as logical "and" $(\wedge)$, logical "or" $(\vee)$, negation $(\neg)$, implication $(\Rightarrow)$, equivalence $(\Leftrightarrow)$ again leads to a complementarity condition of a GLCP.

First we introduce the so-called "augmented GLCP". Consider a GLCP defined by

$$
\begin{aligned}
u & \geq 0 \\
Z u & =0 \\
\sum_{j=1}^{m} \prod_{i \in \phi_{j}} u_{i} & =0
\end{aligned}
$$

with $Z \in \mathbb{R}^{p \times n}$ and $\phi_{1}, \phi_{2}, \ldots, \phi_{m} \subseteq\{1,2, \ldots, n\}$. We call the $u_{i}$ 's the basic variables. Now we introduce some extra variables that will be called mirror variables: we define a vector $v \in \mathbb{R}^{n}$ such that $v_{i}$ is equal to 0 if and only if $u_{i}$ is different from zero. It is easy to verify that this condition is equivalent to the following equations for each $i \in\{1,2, \ldots, n\}$ :

$$
\begin{aligned}
v_{i} & \geq 0 \\
u_{i}+v_{i} & \geq 1 \\
u_{i} v_{i} & =0
\end{aligned} .
$$

Since $u_{i}$ and $v_{i}$ are nonnegative for each $i$, the conditions $u_{i} v_{i}=0$ for $i=$ $1,2, \ldots, n$ are equivalent to the condition

$$
\sum_{i=1}^{n} u_{i} v_{i}=0
$$

which is a condition of the form (16). Since $u_{i}$ and $v_{i}$ are nonnegative for all $i, \sum_{j=1}^{m} \prod_{i \in \phi_{j}} u_{i}$ and $\sum_{i=1}^{n} u_{i} v_{i}$ are also nonnegative. Since a sum of two nonnegative numbers is equal to 0 if and only if each term is equal to 0 , conditions (16) and (20) lead to

$$
\sum_{j=1}^{m} \prod_{i \in \phi_{j}} u_{i}+\sum_{i=1}^{n} u_{i} v_{i}=0 .
$$


This is again a condition of the form (16).

Clearly, equations of the form (18) fit in the ELCP framework. They can also be transformed into GLCP equations by introducing nonnegative slack variables $s_{1}, s_{2}, \ldots, s_{n}$ with $s_{i}=u_{i}+v_{i}-1$ and a nonnegative homogenization parameter $\alpha$. Then we get

$$
u_{i}+v_{i}-s_{i}-\alpha=0
$$

for $i=1,2, \ldots, n$.

So now we have again a GLCP with basic variables $u_{i}$, mirror variables $v_{i}$, slack variables $s_{i}$ and a homogenization parameter $\alpha$. This GLCP will be called the augmented GLCP that corresponds to the GLCP defined by (14)-(16).

Let $k, l \in \mathbb{N}$ and let $\phi_{1}, \phi_{2}, \ldots, \phi_{k}, \psi_{1}, \psi_{2}, \ldots, \psi_{l} \subseteq\{1,2, \ldots, n\}$. Consider the following expressions:

$$
\begin{aligned}
c_{1} & =\sum_{j=1}^{k} \prod_{i \in \phi_{j}} u_{i} \\
c_{2} & =\sum_{j=1}^{l} \prod_{i \in \psi_{j}} u_{i} .
\end{aligned}
$$

Since $u_{i} \geq 0$ for all $i$ we have $c_{1}, c_{2} \geq 0$. Note that the expressions $c_{1}=0$ and $c_{2}=0$ can be considered as complementarity conditions of the form (16).

Lemma 1 The logical "and" of two complementarity conditions of a GLCP leads to a complementarity condition of the given GLCP.

PROOF. Consider a GLCP defined by (14) - (15) and consider two complementarity conditions that are given by $c_{1}=0$ and $c_{2}=0$ with $c_{1}$ and $c_{2}$ defined by (21) - (22) .

Since $c_{1}, c_{2} \geq 0$, we have

$$
\left(c_{1}=0\right) \wedge\left(c_{2}=0\right)
$$

if and only if

$$
c_{1}+c_{2}=0
$$


or equivalently

$$
c_{1}+c_{2}=\sum_{j=1}^{m} \prod_{i \in \gamma_{j}} u_{i}=0
$$

with $m=k+l$ and

$$
\gamma_{j}=\left\{\begin{array}{l}
\phi_{j} \quad \text { for } j=1,2, \ldots, k \\
\psi_{j-k} \text { for } j=k+1, k+2, \ldots, k+l .
\end{array}\right.
$$

Hence, the logical "and" of two complementarity conditions of the form (16) leads again to a complementarity condition of the form (16).

Lemma 2 The logical "or" of two complementarity conditions of a GLCP leads to a complementarity condition of the given GLCP.

PROOF. Consider a GLCP defined by (14) - (15) and consider two complementarity conditions that are given by $c_{1}=0$ and $c_{2}=0$ with $c_{1}$ and $c_{2}$ defined by $(21)-(22)$.

We have

$$
\left(c_{1}=0\right) \vee\left(c_{2}=0\right)
$$

if and only if

$$
c_{1} c_{2}=0
$$

If we define $p=k l$ and sets $\delta_{1}, \delta_{2}, \ldots, \delta_{p}$, such that

$$
\delta_{i+k(j-1)}=\phi_{i} \cup \psi_{j} \quad \text { for } i=1,2, \ldots, k \text { and } j=1,2, \ldots, l \text {, }
$$

we obtain

$$
c_{1} c_{2}=\sum_{j=1}^{p} \prod_{i \in \delta_{j}} u_{i}=0 .
$$

Hence, the logical "or" of two complementarity conditions of the form (16) leads again to a complementarity condition of the form (16). 
Lemma 3 The negation of a complementarity condition of a GLCP leads to a complementarity condition of the augmented GLCP that corresponds to the given GLCP.

PROOF. Consider a GLCP defined by (14) - (15) and the complementarity condition $c_{1}=0$ with $c_{1}$ defined by (21). Now consider the negation of this complementarity condition. Since $u_{i} \geq 0$ for all $i$, we have $\neg\left(c_{1}=0\right)$ or

$$
\sum_{j=1}^{m} \prod_{i \in \phi_{j}} u_{i} \neq 0
$$

if and only if

$$
\exists j \in\{1,2, \ldots, m\} \text { such that } \prod_{i \in \phi_{j}} u_{i} \neq 0
$$

or

$$
\exists j \in\{1,2, \ldots, m\} \text { such that } \forall i \in \phi_{j}: u_{i} \neq 0
$$

If we use the mirror variables $v_{i}$, this condition leads to

$$
\exists j \in\{1,2, \ldots, m\} \text { such that } \forall i \in \phi_{j}: v_{i}=0
$$

or

$$
\exists j \in\{1,2, \ldots, m\} \text { such that } \sum_{i \in \phi_{j}} v_{i}=0
$$

since $v_{i} \geq 0$ for all $i$. Hence, we have $\neg\left(c_{1}=0\right)$ if and only if

$$
\prod_{j=1}^{m} \sum_{i \in \phi_{j}} v_{i}=0
$$

If we write out the multiplications and additions in this condition we obtain

$$
\sum_{j=1}^{q} \prod_{i \in \eta_{j}} v_{i}=0
$$

with $q=\left(\# \phi_{1}\right)\left(\# \phi_{2}\right) \ldots\left(\# \phi_{m}\right)$ and

$$
\left\{\eta_{j} \mid j=1,2, \ldots, q\right\}=\left\{\left\{i_{1}, i_{2}, \ldots, i_{m}\right\} \mid i_{1} \in \phi_{1}, \ldots, i_{m} \in \phi_{m}\right\} .
$$


Clearly, (23) is a complementarity condition of the form (16) of the augmented GLCP that corresponds to the given GLCP.

Lemma 4 Combining two complementarity conditions of a GLCP by implication or equivalence leads to a complementarity condition of the augmented GLCP that corresponds to the given GLCP.

PROOF. This is a direct consequence of Lemmas 1, 2 and 3, and the fact that if $P$ and $Q$ are logical expressions, we have

$$
\begin{array}{lll}
P \Rightarrow Q \quad \text { if and only if } & (\neg P) \vee Q \\
P \Leftrightarrow Q \quad \text { if and only if } & (P \wedge Q) \vee((\neg P) \wedge(\neg Q)) .
\end{array}
$$

In a similar way we can prove that more complex nested logical combinations of complementarity conditions of the form (16) lead again to a complementarity condition of the form (16). As a consequence, we have:

Proposition 5 Composing complementarity conditions of a GLCP by nested combinations of logical operators such as logical "and", logical "or", negation, implication or equivalence leads to a complementarity condition of the augmented GLCP that corresponds to the given GLCP.

It is easy to verify that we can also apply a similar reasoning for complementarity conditions of ELCPs:

Proposition 6 Composing complementarity conditions of an ELCP by nested combinations of logical operators such as logical "and", logical "or", negation, implication or equivalence leads again to a complementarity condition of the form (12).

\subsection{The LDCP is a special case of the ELCP and the GLCP}

Now we come to the main result of this paper:

Theorem 7 The LDCP is a special case of the GLCP. 
PROOF. Consider the LDCP defined by (6)-(10). If we define

$$
y=\left[\begin{array}{c}
y_{0} \\
y_{1} \\
\vdots \\
y_{n-1}
\end{array}\right], u=\left[\begin{array}{c}
u_{0} \\
u_{1} \\
\vdots \\
u_{n-1}
\end{array}\right], q=\left[\begin{array}{c}
C x_{0} \\
C A x_{0} \\
\vdots \\
C A^{n-1} x_{0}
\end{array}\right]
$$

and

$$
P=\left[\begin{array}{ccccc}
D & O & O & \ldots & O \\
C B & D & O & \ldots & O \\
\vdots & \vdots & \vdots & \ddots & \vdots \\
C A^{n-2} B & C A^{n-3} B & C A^{n-4} B & \ldots & D
\end{array}\right],
$$

then we have $y=P u+q$.

Consider the sign decomposition of $y$ and $u$ :

$$
\begin{gathered}
y=y^{+}-y^{-} \\
u=u^{+}-u^{-} \\
y^{+}, y^{-}, u^{+}, u^{-} \geq 0 \\
\left(y^{+}\right)^{T} y^{-}=0 \text { and }\left(u^{+}\right)^{T} u^{-}=0 .
\end{gathered}
$$

The last condition is equivalent to $\left(y^{+}\right)^{T} y^{-}+\left(u^{+}\right)^{T} u^{-}=0$ by Lemma 1 . Now we define

$$
x=\left[\begin{array}{l}
y^{+} \\
u^{+} \\
y^{-} \\
u^{-}
\end{array}\right]
$$

and we introduce a nonnegative real number $\alpha$ that will be used to homogenize the problem (cf. 3.4.2). If we define

$$
Z=\left[\begin{array}{llll}
I & -P & -I & P
\end{array}\right]
$$

then we have 


$$
\begin{aligned}
& {\left[\begin{array}{ll}
Z-q
\end{array}\right]\left[\begin{array}{l}
x \\
\alpha
\end{array}\right]=0} \\
& {\left[\begin{array}{l}
x \\
\alpha
\end{array}\right] \geq 0} \\
& \sum_{i=1}^{2 n k} x_{i} x_{i+2 n k}=0 \text {. }
\end{aligned}
$$

Now we show that combining condition (26) with the complementarity conditions (9) - (10) of the LDCP results in a complementarity condition of the form (11). We shall do this for the case with $n=2$ and $k=1$. The general case can be dealt with in a similar way.

Consider condition (9) for $i=1$. For ease of notation we shall drop the index $i$. Now we show that the condition

$$
\left[\begin{array}{ll}
y_{0} & y_{1}
\end{array}\right]^{T}=0 \quad \text { and } \quad\left[u_{0} u_{1}\right]^{T} \succeq 0
$$

leads to a logical combination of a number of complementarity conditions. Clearly, the first part of this condition is equivalent to $y_{0}^{+}=y_{0}^{-}=y_{1}^{+}=y_{1}^{-}=0$. Since $y_{0}^{+}, y_{0}^{-}, y_{1}^{+}, y_{1}^{-} \geq 0$, this condition is equivalent to

$$
y_{0}^{+}+y_{0}^{-}+y_{1}^{+}+y_{1}^{-}=0 \text {. }
$$

Note that we could also consider (28) as the logical "and" of the elementary complementarity conditions $y_{0}^{+}=0, y_{0}^{-}=0, y_{1}^{+}=0, y_{1}^{-}=0$.

The second part of condition (27) is equivalent to

$$
\left(u_{0} \geq 0\right) \wedge\left(\left(u_{0}=0\right) \Rightarrow\left(u_{1} \geq 0\right)\right) \text {, }
$$

which can be rewritten as

$$
\left(u_{0}^{-}=0\right) \wedge\left(\left(\left(u_{0}^{+}=0\right) \wedge\left(u_{0}^{-}=0\right)\right) \Rightarrow\left(u_{1}^{-}=0\right)\right) .
$$

This condition is a logical combination of elementary complementarity conditions and can be rewritten as one complementarity condition of the form (11) by Proposition 5 .

This implies that the complementarity conditions of the LDCP together with condition (26) lead to one large complementarity condition of the form (11). 
So we finally get a GLCP. Any solution of this GLCP that has a nonzero $\alpha$ component will - after normalization of the $\alpha$ component to 1 and extraction of the vectors $y_{0}, y_{1}, \ldots, y_{n-1}, u_{0}, u_{1}, \ldots, u_{n-1}$ - yield a solution of the LDCP. So we can say that the LDCP is a special case of the GLCP.

Remark 8 It is easy to verify that we shall always have $\left(y_{0}^{-}\right)_{i}=\left(u_{0}^{-}\right)_{i}=0$ for all $i$.

Corollary 9 The $L D C P$ is a special case of the ELCP.

PROOF. This is a direct consequence of Theorem 7 and the fact that the GLCP is a special case of the ELCP.

Remark 10 Consider the GLCP that corresponds to the LDCP of the proof of Theorem 7. Assume that the vector $\tilde{x} \in \mathbb{R}^{4 n k}$ contains the mirror variables that correspond to the basic variables of this GLCP and that the vector $s \in \mathbb{R}^{4 n k}$ contains the slack variables of this GLCP. If we remove the slack variables s, we get an ELCP of the following form:

$$
\begin{aligned}
I w & \geq 0 \\
{\left[\begin{array}{ll}
I & I
\end{array}\right] w } & \geq\left[\begin{array}{l}
1 \\
1 \\
\vdots \\
1
\end{array}\right] \\
{\left[\begin{array}{ll}
Z & O
\end{array}\right] w } & =q \\
\sum \prod(I w)_{i} & =0
\end{aligned}
$$

where $w=\left[\begin{array}{cc}x^{T} & \tilde{x}^{T}\end{array}\right]^{T}$. Note that the ELCP defined above has $4 k n$ variables less than the GLCP. This is important since in general the execution time of the ELCP/GLCP algorithms of [4,6,7] increases exponentially as the number of variables increases (see also Section 3.4.3).

In the particular case of an ELCP that corresponds to an LDCP, the effect of exponentially increasing execution times is counteracted by the fact that in that case the complementarity condition will contain many terms, which will result in a large reduction of intermediate solutions and thus also in a reduction of the execution time of the algorithm (see [6]). 


\section{An example}

Let

$$
\begin{aligned}
& A=\left[\begin{array}{rr}
-2 & -3 \\
5 & -5
\end{array}\right], \quad B=\left[\begin{array}{rr}
5 & 5 \\
-2 & -3
\end{array}\right], C=\left[\begin{array}{l}
2-2 \\
3-4
\end{array}\right], \\
& D=\left[\begin{array}{rr}
-1 & 3 \\
-1 & -1
\end{array}\right], \quad x_{0}=\left[\begin{array}{l}
4 \\
0
\end{array}\right]
\end{aligned}
$$

and consider the following LDCP:

Find $y_{0}, y_{1}, u_{0}, u_{1} \in \mathbb{R}^{2}$ such that

$$
\begin{aligned}
& y_{0}=C x_{0}+D u_{0} \\
& y_{1}=C A x_{0}+C B u_{0}+D u_{1}
\end{aligned}
$$

and such that at least one of the following statements is true for each index $i \in\{1,2\}$ :

$$
\begin{aligned}
& {\left[\begin{array}{ll}
\left(y_{0}\right)_{i} & \left(y_{1}\right)_{i}
\end{array}\right]^{T}=0 \text { and }\left[\begin{array}{ll}
\left(u_{0}\right)_{i} & \left(u_{1}\right)_{i}
\end{array}\right]^{T} \succeq 0} \\
& {\left[\begin{array}{lll}
\left(y_{0}\right)_{i} & \left(y_{1}\right)_{i}
\end{array}\right]^{T} \succeq 0 \text { and }\left[\begin{array}{ll}
\left(u_{0}\right)_{i} & \left(u_{1}\right)_{i}
\end{array}\right]^{T}=0}
\end{aligned}
$$

If we use the reasoning of Section 4.2 then we find that this LDCP is equivalent to the following ELCP:

Find $x, \tilde{x} \in \mathbb{R}^{16}$ such that

$$
\begin{aligned}
& x, \tilde{x} \geq 0 \\
& x_{i}+\tilde{x}_{i} \geq 1 \text { for } i=1,2, \ldots, 16 \\
& {\left[\begin{array}{rrrrrrrrrrrrrrrr}
1 & 0 & 0 & 0 & 1 & -3 & 0 & 0 & -1 & 0 & 0 & 0 & -1 & 3 & 0 & 0 \\
0 & 1 & 0 & 0 & 1 & 1 & 0 & 0 & 0 & -1 & 0 & 0 & -1 & -1 & 0 & 0 \\
0 & 0 & 1 & 0 & -14 & -16 & 1 & -3 & 0 & 0 & -1 & 0 & 14 & 16 & -1 & 3 \\
0 & 0 & 0 & 1 & -23 & -27 & 1 & 1 & 0 & 0 & 0 & -1 & 23 & 27 & -1 & -1
\end{array}\right] x=\left[\begin{array}{r}
8 \\
12 \\
-56 \\
-104
\end{array}\right]} \\
& x_{1} x_{5}+x_{1} x_{7}+x_{1} x_{15}+x_{1} \tilde{x}_{1}+x_{2} x_{6}+x_{2} x_{8}+ \\
& x_{2} x_{16}+x_{2} \tilde{x}_{2}+x_{3} x_{5}+x_{3} x_{7}+x_{3} x_{11}+x_{3} x_{15}+ \\
& x_{3} \tilde{x}_{1} \tilde{x}_{9}+x_{3} \tilde{x}_{3}+x_{4} x_{6}+x_{4} x_{8}+x_{4} x_{12}+x_{4} x_{16}+
\end{aligned}
$$




$$
\begin{array}{r}
x_{4} \tilde{x}_{2} \tilde{x}_{10}+x_{4} \tilde{x}_{4}+x_{5} x_{11}+x_{5} \tilde{x}_{5}+x_{6} x_{12}+x_{6} \tilde{x}_{6}+ \\
x_{7} x_{11}+x_{7} x_{15}+x_{7} \tilde{x}_{7}+x_{8} x_{12}+x_{8} x_{16}+x_{8} \tilde{x}_{8}+x_{9}+ \\
x_{10}+x_{11} x_{15}+x_{11} \tilde{x}_{1} \tilde{x}_{9}+x_{11} \tilde{x}_{11}+x_{12} x_{16}+x_{12} \tilde{x}_{2} \tilde{x}_{10}+ \\
x_{12} \tilde{x}_{12}+x_{13}+x_{14}+x_{15} \tilde{x}_{5} \tilde{x}_{13}+x_{15} \tilde{x}_{15}+ \\
x_{16} \tilde{x}_{6} \tilde{x}_{14}+x_{16} \tilde{x}_{16}=0 .
\end{array}
$$

If we solve this ELCP using the algorithm of $[6,7]$ we find that there are 4 different possible solutions:

$$
\begin{aligned}
& y_{0}=\left[\begin{array}{r}
8 \\
12
\end{array}\right], \quad y_{1}=\left[\begin{array}{r}
-56 \\
-104
\end{array}\right], \quad u_{0}=\left[\begin{array}{l}
0 \\
0
\end{array}\right], \quad u_{1}=\left[\begin{array}{l}
0 \\
0
\end{array}\right], \\
& y_{0}=\left[\begin{array}{r}
44 \\
0
\end{array}\right], y_{1}=\left[\begin{array}{r}
796 \\
0
\end{array}\right], \quad u_{0}=\left[\begin{array}{r}
0 \\
12
\end{array}\right], \quad u_{1}=\left[\begin{array}{r}
0 \\
220
\end{array}\right], \\
& y_{0}=\left[\begin{array}{l}
0 \\
0
\end{array}\right], y_{1}=\left[\begin{array}{l}
0 \\
0
\end{array}\right], u_{0}=\left[\begin{array}{r}
11 \\
1
\end{array}\right], \quad u_{1}=\left[\begin{array}{r}
160.5 \\
15.5
\end{array}\right]
\end{aligned}
$$

and

$$
y_{0}=\left[\begin{array}{l}
0 \\
4
\end{array}\right], \quad y_{1}=\left[\begin{array}{r}
0 \\
24
\end{array}\right], \quad u_{0}=\left[\begin{array}{l}
8 \\
0
\end{array}\right], \quad u_{1}=\left[\begin{array}{r}
56 \\
0
\end{array}\right] .
$$

Since the LDCP considered here has more than one solution, the associated mode selection problem will not have a unique solution either. For more information on the connection between the mode selection problem for complementary-slackness systems and the LDCP and for some worked examples the interested reader is referred to $[13-15,17]$.

\section{The ELCP and hybrid systems}

Hybrid systems arise from the interaction between discrete event systems and continuous variable systems (these are systems that can be modeled using difference or differential equations). In general we could say that a hybrid system can be in one of several "regimes" whereby in each regime the behavior of the system can be described by a system of difference or differential equations, and that the system switches from one regime to another due to the occurrence of events. 
In $[6,9]$ we have shown that the ELCP can be used to solve some basic problems that appear in the max-plus-algebraic system theory for discrete event systems. In $[13-15,17]$ it has been shown that the LDCP and the GLCP (both of which are special cases of the ELCP) play a role in the modeling and analysis of continuous variable systems and systems with discontinuous dynamics, both of which can be considered as special classes of hybrid systems. In $[10,11]$ we have used the ELCP in a model that describes the evolution of the queue lengths at a traffic-light controlled intersection, which can also be considered as a special type of hybrid system. Since the class of discrete event systems can also be considered as a subclass of the class of hybrid systems, this seems to indicate that the ELCP will play an important role in many analysis problems for hybrid systems.

Note that the main purpose of this paper is to show that the LDCP is a special case of the ELCP and that the ELCP can be considered as a general framework for the analysis of special classes of hybrid systems. We certainly do not claim that the ELCP approach is more efficient than, e.g., the use of the LDCP in the analysis of complementary-slackness systems. Furthermore, it is obvious that each class of hybrid systems that can be analyzed using the ELCP will lead to a special case of the ELCP that is especially suited to the class of systems under consideration. The computational complexity of these ELCPs and the development of efficient algorithms to solve them or to determine whether their solution set is non-empty or a singleton still are open problems. One possible approach to tackle the complexity problem is to use approximations and/or to develop procedures to efficiently obtain suboptimal solutions (see, e.g., [10]).

\section{Conclusions and topics for further research}

In this paper we have shown that the Linear Dynamic Complementarity Problem (LDCP) can be considered as a special case of the Generalized Linear Complementarity Problem (GLCP) and of the Extended Linear Complementarity Problem (ELCP).

Topics for further research include: development of efficient algorithms to compute solutions of (special cases of) the ELCP, development of algorithms to determine whether an ELCP, a GLCP or an LDCP has a (unique) solution, development of approximation algorithms or procedures to find suboptimal solutions, and investigation of the possible applications of (special cases of) the ELCP in the analysis of hybrid systems. 


\section{Acknowledgements}

This research was sponsored by the Concerted Action Project of the Flemish Community, entitled "Model-based Information Processing Systems" (GOAMIPS), by the Belgian program on interuniversity attraction poles (IUAP P4-02 and IUAP P4-24), by the ALAPEDES ("Algebraic Approach to Performance Evaluation of Discrete Event Systems") project of the European Community Training and Mobility of Researchers Program, and by the European Commission Human Capital and Mobility Network SIMONET ("System Identification and Modelling Network").

\section{References}

[1] F. Baccelli, G. Cohen, G.J. Olsder, and J.P. Quadrat. Synchronization and Linearity. John Wiley \& Sons, New York, 1992.

[2] R.W. Cottle, J.S. Pang, and R.E. Stone. The Linear Complementarity Problem. Academic Press, Boston, 1992.

[3] R.A. Cuninghame-Green. Minimax Algebra, volume 166 of Lecture Notes in Economics and Mathematical Systems. Springer-Verlag, Berlin, Germany, 1979.

[4] B. De Moor. Mathematical Concepts and Techniques for Modelling of Static and Dynamic Systems. PhD thesis, Faculty of Applied Sciences, K.U.Leuven, Leuven, Belgium, June 1988.

[5] B. De Moor, L. Vandenberghe, and J. Vandewalle. The generalized linear complementarity problem and an algorithm to find all its solutions. Mathematical Programming, 57:415-426, 1992.

[6] B. De Schutter. Max-Algebraic System Theory for Discrete Event Systems. PhD thesis, Faculty of Applied Sciences, K.U.Leuven, Leuven, Belgium, February 1996.

[7] B. De Schutter and B. De Moor. The extended linear complementarity problem. Mathematical Programming, 71(3):289-325, December 1995.

[8] B. De Schutter and B. De Moor. Minimal realization in the max algebra is an extended linear complementarity problem. Systems \&3 Control Letters, 25(2):103-111, May 1995.

[9] B. De Schutter and B. De Moor. A method to find all solutions of a system of multivariate polynomial equalities and inequalities in the max algebra. Discrete Event Dynamic Systems: Theory and Applications, 6(2):115-138, March 1996.

[10] B. De Schutter and B. De Moor. Optimal traffic light control for a single intersection. Technical Report 96-90, ESAT-SISTA, K.U.Leuven, Leuven, Belgium, December 1996. Submitted for publication. 
[11] B. De Schutter and B. De Moor. Optimal traffic light control for a single intersection. In Proceedings of the 1997 International Symposium on Nonlinear Theory and its Applications (NOLTA'97), Hawaii, USA, Nov. 29-Dec. 3, 1997.

[12] M.C. Ferris and J.S. Pang, editors. Complementarity and Variational Problems: State of the Art. Philadelphia, Pennsylvania: SIAM, 1997. (Proceedings of the International Conference on Complementarity Problems, Baltimore, Maryland, USA, November 1995).

[13] W.P.M.H. Heemels, J.M. Schumacher, and S. Weiland. Linear complementarity systems. Technical Report 97 I/01, Department of Electrical Engineering, Eindhoven University of Technology, Eindhoven, The Netherlands, July 1997.

[14] J.M. Schumacher. Some modeling aspects of unilaterally constrained dynamics. In Proceedings of the ESA International Workshop on Advanced Mathematical Methods in the Dynamics of Flexible Bodies, ESA-ESTEC, Noordwijk, The Netherlands, June 1996.

[15] A.J. van der Schaft and J.M. Schumacher. Complementary modeling of hybrid systems. Technical Report BS-R9611, CWI, Amsterdam, The Netherlands, 1996.

[16] A.J. van der Schaft and J.M. Schumacher. Hybrid systems modelling and complementarity problems. In Proceedings of the European Control Conference (ECC'97), Brussels, Belgium, paper 868, July 1997.

[17] L. Vandenberghe, B. De Moor, and J. Vandewalle. The generalized linear complementarity problem applied to the complete analysis of resistive piecewiselinear circuits. IEEE Transactions on Circuits and Systems, 36(11):1382-1391, November 1989. 